\title{
Fluctuation relations for a driven Brownian particle
}

\author{
A. Imparato and L. Peliti \\ Dipartimento di Scienze Fisiche, INFN-Sezione di Napoli, CNISM-Sezione di Napoli \\ Università “Federico II”, Complesso Monte S. Angelo, I-80126 Napoli (Italy)
}

\begin{abstract}
We consider a driven Brownian particle, subject to both conservative and non-conservative applied forces, whose probability evolves according to the Kramers equation. We derive a general fluctuation relation, expressing the ratio of the probability of a given Brownian path in phase space with that of the time-reversed path, in terms of the entropy flux to the heat reservoir. This fluctuation relation implies those of Seifert, Jarzynski and Gallavotti-Cohen in different special cases.
\end{abstract}

PACS numbers: 05.40.-a,05.70.Ln

\section{INTRODUCTION}

Fluctuation relations of increasing generality have been derived in the recent years, starting from the Evans-Searles relation [1]]

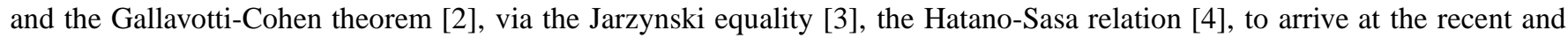
rather general Seifert relation [5], from which the previous identities can be derived by simple manipulations. These results have been obtained by considering situations of increasing generality, and it appears that a sufficient condition for fluctuation relations to hold is the existence of mechanical equilibrium at each instant of time [6]. However, this condition is not necessary, as shown by the derivation by Kurchan [7] of some fluctuation relations for a Brownian particle with inertia.

We wish to point out that it is possible to obtain a compact derivation of the fluctuation relations by deriving a key relation between the probability of a path in phase space and that of its time-reversed image, for a particle with inertia. This relation encompasses the relation derived by Crooks [8] for a particle subject to conservative forces and extended by Seifert [5] to arbitrary forces in the overdamped regime. Our derivation is inspired by Kurchan's one [7], but is made more compact and more general by considering the generating functional of the conditional probabilities for the paths.

As a bonus, we obtain an explicit expression for the entropy production and the heat flow for a Brownian particle with inertia, which generalizes the expression introduced by Sekimoto [9], valid in the overdamped regime.

The key relation between the probability of a path $\omega$, conditioned on its initial point, and that of the reverse path $\tilde{\omega}$, conditioned on its initial point, is derived in sec. II. The relation involves a functional of the path, whose physical interpretation as the entropy flow into the heat bath is given in sec. III. In sec. IV, using the formalism previously introduced, the explicit expression of path probability as a form of the Onsager-Machlup functional is recovered, and the fluctuation relation is shown to hold for such a functional. A brief discussion follows, while the derivation of the relation between the time derivatives of "global" and "local" entropies is given in the Appendix.

\section{A GENERALIZED CROOKS IDENTITY}

We consider a particle in $d$ dimensions, whose evolution is described by the Langevin equation

$$
m \ddot{\boldsymbol{r}}=-\zeta \dot{\boldsymbol{r}}-\frac{\partial U_{\mu}(\boldsymbol{r})}{\partial \boldsymbol{r}}+\boldsymbol{f}_{\mu}+\boldsymbol{\eta}(t),
$$

where $\mu$ is a time-dependent parameter, and $f_{\mu}$ is a non conservative force. We assume that $\boldsymbol{\eta}(t)$ is a delta-correlated white noise with variance $2 \zeta T$. Boltzmann's constant is set equal to 1 throughout.

We denote by $x=(\boldsymbol{p}, \boldsymbol{r})$ the microstate, and by $P(x, t)$ the probability distribution function (pdf) of the process (1) in phase space. This function evolves according to the differential equation

$$
\frac{\partial P(x, t)}{\partial t}=\mathcal{L}_{\mu(t)} P(x, t)
$$

${ }^{*}$ Present address: Dipartimento di Fisica, Politecnico di Torino, C. Duca degli Abruzzi 24, 10129 Torino (Italy). 
where $\mathcal{L}_{\mu}$ is the Kramers operator

$$
\mathcal{L}_{\mu} P=\frac{\partial}{\partial \boldsymbol{r}} \cdot\left(-\frac{\boldsymbol{p}}{m} P\right)+\frac{\partial}{\partial \boldsymbol{p}} \cdot\left[\left(\zeta \frac{\boldsymbol{p}}{m}+\frac{\partial U_{\mu}}{\partial \boldsymbol{r}}-\boldsymbol{f}_{\mu}\right) P+\zeta T \frac{\partial P}{\partial \boldsymbol{p}}\right] .
$$

Let us define the time-reversal operator $\mathcal{I}$ by

$$
\mathcal{I}(\boldsymbol{p}, \boldsymbol{r})=(-\boldsymbol{p}, \boldsymbol{r})
$$

and the associated operator $Q_{t}$ by

$$
Q_{t} \psi(x)=\mathrm{e}^{-E(x, t) / T} \psi(\mathcal{I} x)
$$

We assume that the system energy, defined by

$$
E(x, t)=\sum_{i=1}^{d} \frac{p_{i}^{2}}{2 m}+U_{\mu(t)}(\boldsymbol{r})
$$

is invariant under time inversion:

$$
E(\mathcal{I} x, t)=E(x, t) .
$$

The microscopic reversibility of the process (1) implies the following relation

$$
Q_{t}^{-1} \mathcal{L}_{\mu(t)} Q_{t}=\mathcal{L}_{\mu(t)}^{\dagger}+\frac{\boldsymbol{f}_{\mu(t)}}{T} \cdot \frac{\boldsymbol{p}}{m}
$$

In the following the symbol $\omega$ will indicate an arbitrary path in the system phase space:

$$
\omega: \quad t \stackrel{\omega}{\longrightarrow} x(t) .
$$

For any arbitrary function $a(x, t)$, and any arbitrary path $\omega$ in the system phase space we define the quantity $A\left(t, t_{0}, \omega\right)$ as the integral of $a(x, t)$ over the interval $\left[t_{0}, t\right]$ along the given path $\omega$ :

$$
A\left(t, t_{0}, \omega\right) \equiv \int_{t_{0}}^{t} \mathrm{~d} t^{\prime} a\left(x\left(t^{\prime}\right), t^{\prime}\right)
$$

The joint probability distribution function $\Phi(x, A, t)$ evolves according to the differential equation

$$
\frac{\partial \Phi}{\partial t}=\mathcal{L}_{\mu(t)} \Phi-a(x, t) \frac{\partial \Phi}{\partial A}
$$

Let us define the function

$$
\begin{aligned}
\Psi\left(x, t ; x_{0}, t_{0} ; \mu, a\right) & =\int_{x\left(t_{0}\right)=x_{0}}^{x(t)=x} \mathcal{D} \omega \mathcal{P}(\omega) \int \mathrm{d} A \delta\left(A-A\left(t, t_{0}, \omega\right)\right) \mathrm{e}^{A\left(t, t_{0}, \omega\right)} \\
& =\int \mathrm{d} A \mathrm{e}^{A} \Phi(x, A, t),
\end{aligned}
$$

where we make explicit the fact that $\Psi$ depends on the initial condition $x_{0}, t_{0}$, and is a functional of $\mu(t)$ and $a(x, t)$. Then $\Psi$ satisfies the following equation:

$$
\frac{\partial \Psi}{\partial t}=\mathcal{L}_{\mu(t)} \Psi+a(x, t) \Psi .
$$

A number of fluctuation relations can be easily derived from this expression. To illustrate this point, we report here the derivation of the Hatano-Sasa relation [П]. Let

$$
P^{\mathrm{SS}}(x, \mu)=\mathrm{e}^{-\phi(x, \mu)}
$$

be the steady-state distribution function associated to the operator $\mathcal{L}_{\mu}$, which satisfies

$$
\mathcal{L}_{\mu} P^{\mathrm{SS}}(x, \mu)=0
$$


Let us choose the arbitrary function $a(x, t)$ as follows:

$$
a^{*}(x, t)=\dot{\mu}(t) \frac{\partial \ln P^{S S}}{\partial \mu}=-\dot{\mu}(t) \frac{\partial \phi}{\partial \mu},
$$

so that eq. (10) reads

$$
A=-\left.\int_{t_{0}}^{t} \mathrm{~d} t^{\prime} \dot{\mu}\left(t^{\prime}\right) \frac{\partial \phi}{\partial \mu}\right|_{x\left(t^{\prime}\right), \mu\left(t^{\prime}\right)}
$$

Using the definition of $\Psi$, eq. (12), one finds

$$
\left\langle\delta(x-x(t)) \mathrm{e}^{A(t)}\right\rangle=\int \mathrm{d} A \mathrm{e}^{A} \Phi(x, A, t)=\Psi\left(x, t ; x_{0}, t_{0} ; \mu, a^{*}\right) .
$$

But $\Psi$ is solution of eq. (13), which for the particular choice $a=a^{*}$ takes the form

$$
\frac{\partial \Psi}{\partial t}=\mathcal{L}_{\mu(t)} \Psi-\dot{\mu} \frac{\partial \phi}{\partial \mu} \Psi .
$$

The solution of this last equation, satisfying the initial condition $\Psi\left(x, t_{0}\right)=P^{\mathrm{SS}}\left(x, \mu\left(t_{0}\right)\right)$, is $\Psi=\mathrm{e}^{-\phi(x, \mu(t))}=P^{\mathrm{SS}}(x, \mu(t))$, and thus eq. (18) becomes

$$
\left\langle\delta(x-x(t)) \mathrm{e}^{A(t)}\right\rangle=P^{\mathrm{SS}}(x, \mu(t))
$$

which is the Hatano-Sasa identity, and reduces to the Jarzynski equality when the non-conservative force $f_{\mu}$ vanishes throughout.

Now, let the functional $\widetilde{\Psi}\left(x, t ; x_{0}, t_{0} ; \mu, a\right)$ be defined by

$$
\widetilde{\Psi}\left(x, t ; x_{0}, t_{0} ; \mu, a\right) \equiv Q_{t}^{-1} \Psi\left(x, t ; x_{0}, t_{0} ; \mu, a\right) Q_{t_{0}} .
$$

One can easily check that $\widetilde{\Psi}$ satisfies the evolution equation

$$
\frac{\partial \widetilde{\Psi}}{\partial t}=\mathcal{L}_{t}^{\dagger} \widetilde{\Psi}+\left[\frac{1}{T} \frac{\partial E}{\partial t}+\frac{f_{\mu(t)}}{T} \cdot \frac{p}{m}+a(\mathcal{I} x, t)\right] \widetilde{\Psi} .
$$

Using equations (5) and (12), we obtain the following equality

$$
\widetilde{\Psi}\left(\mathcal{I} x, t ; \mathcal{I} x_{0}, t_{0} ; \mu, a\right)=\mathrm{e}^{\left[E(x, t)-E\left(x_{0}, t_{0}\right)\right] / T} \Psi\left(x, t ; x_{0}, t_{0} ; \mu, a\right) .
$$

Furthermore, upon integration of eq. (22), we obtain

$$
\widetilde{\Psi}\left(\mathcal{I} x, t ; \mathcal{I} x_{0}, t_{0} ; \mu, a\right)=\Psi\left(\mathcal{I} x_{0}, t ; \mathcal{I} x, t_{0} ; \tilde{\mu}, \tilde{a}+w\right),
$$

where we have defined, $\forall t^{\prime} \in\left[t_{0}, t\right]$,

$$
\begin{aligned}
\tilde{t}^{\prime} & =t-\left(t^{\prime}-t_{0}\right) \\
\tilde{\mu}\left(t^{\prime}\right) & =\mu\left(\tilde{t}^{\prime}\right) ; \\
\tilde{a}\left(x, t^{\prime}\right) & =a\left(\mathcal{I} x, \tilde{t}^{\prime}\right) ; \\
w(x, t) & =\frac{1}{T}\left[\frac{\partial E}{\partial t}+\boldsymbol{f}_{\mu(t)} \cdot \frac{\boldsymbol{p}}{m}\right] .
\end{aligned}
$$

Thus, substituting eq. (23) into eq. (24) we obtain

$$
\Psi\left(x, t ; x_{0}, t_{0} ; \mu, a\right)=\Psi\left(\mathcal{I} x_{0}, t ; \mathcal{I} x, t_{0} ; \tilde{\mu}, \tilde{a}+w\right) \mathrm{e}^{-\left[E(x, t)-E\left(x_{0}, t_{0}\right)\right] / T} .
$$

We choose $a(x, t)$ as

$$
a(x, t)=\lambda(t) x=\lambda^{(p)}(t) \cdot \boldsymbol{p}+\boldsymbol{\lambda}^{(r)}(t) \cdot \boldsymbol{r} .
$$

Then, using the definition of $\Psi$, eq. (12), we obtain the generating functional for the path probabilities

$$
\Psi\left(x, t ; x_{0}, t_{0} ; \mu, \lambda x\right)=\int_{x\left(t_{0}\right)=x_{0}}^{x(t)=x} \mathcal{D} \omega \mathcal{P}(\omega) \exp \left(\int_{t_{0}}^{t} \mathrm{~d} t^{\prime} \lambda\left(t^{\prime}\right) x\left(t^{\prime}\right)\right) .
$$


Now, for each path $\omega$, starting from $x_{0}$ and finishing in $x$, the following equality holds

$$
E(x, t)-E\left(x_{0}, t_{0}\right)=\int_{t_{0}}^{t}\left(\frac{\partial E}{\partial t^{\prime}} \mathrm{d} t^{\prime}+\left.\frac{\partial E}{\partial x}\right|_{x\left(t^{\prime}\right)} \mathrm{d} x\left(t^{\prime}\right)\right) .
$$

In this expression, the integral has to be interpreted according to Stratonovich, otherwise additional terms would appear. The relation (31) holds for arbitrary $\lambda$, and thus leads to an analogous relation for the probabilities of the paths $\omega$ conditional on their initial points. Indeed, given the path $\omega$ defined by (9), we formally have

$$
\mathcal{P}\left(\omega, \mu \mid x_{0}, t_{0}\right)=\int \mathcal{D} \lambda \mathrm{e}^{-\int_{t_{0}}^{t} \mathrm{~d} t^{\prime} \lambda\left(t^{\prime}\right) x\left(t^{\prime}\right)} \Psi\left(x, t ; x_{0}, t_{0} ; \mu, \lambda x\right),
$$

where we have introduced the functional integration over $\lambda$ from $-\mathrm{i} \infty$ to $+\mathrm{i} \infty$. Applying this operation to eqs. 29. and (31) we obtain the central result

$$
\mathcal{P}\left(\omega, \mu \mid x_{0}, t_{0}\right)=\mathcal{P}\left(\widetilde{\omega}, \tilde{\mu} \mid \mathcal{I} x, t_{0}\right) \exp \left[\int_{x_{0}}^{x} \frac{\mathrm{d} Q_{2}}{T}\right]
$$

where

$$
\mathrm{d} Q_{2}(t)=-\left.\frac{\partial E}{\partial x}\right|_{x(t)} \mathrm{d} x(t)+\boldsymbol{f}_{\mu(t)} \cdot \frac{\boldsymbol{p}(t)}{m} \mathrm{~d} t .
$$

Thus Crooks's and Seifert's identities ([8, eq. (13)], [5, eq. (14)]) hold also in the case in which the Brownian particle is subject to conservative and nonconservative forces, which do not necessarily equilibrate mechanically with the friction force at each instant in time. Note that the expression of $\mathrm{d} Q_{2}$ must be supplemented by its interpretation as a Stratonovich differential.

Seifert [5] has taught us how to derive the relevant fluctuation relations from the identity (34), by introducing carefully chosen "initial" and "final" pdf's $p_{0}\left(x_{0}\right), p_{1}(\mathcal{I} x)$. For example, let $p_{0}\left(x_{0}\right)$ be an arbitrary normalized initial condition at time $t_{0}$, and $p_{1}(x)$ be the corresponding evolved pdf at time $t$, and define

$$
R=\int_{t_{0}}^{t} \frac{\mathrm{d} Q_{2}}{T}+\ln \frac{p_{0}\left(x_{0}\right)}{p_{1}(x)}
$$

Then

$$
\left\langle\delta(x-x(t)) \mathrm{e}^{-R}\right\rangle=\int \mathrm{d} x_{0} \int \mathcal{D} \omega \mathcal{P}\left(\widetilde{\omega}, \tilde{\mu} \mid \mathcal{I} x, t_{0}\right) p_{1}(x)=p_{1}(x) .
$$

This result can be obtained by changing the integral over $\omega$ into an integral over $\widetilde{\omega}$ and by exploiting the normalization of the conditional probability of the evolution from $\mathcal{I} x$ to $\mathcal{I} x_{0}$ over the final state $\mathcal{I} x_{0}$. Then one can interpret $R$ as the total entropy change, being the sum between the entropy given to the heat bath and the entropy change of the particle, as we shall see in the next section. The Jarzynski equality also follows from eq. (34) by taking for $p_{0}\left(x_{0}\right)$ and $p_{1}(x)$ the equilibrium distributions corresponding to the initial and final states. If one takes instead $\mu$ as a constant, and collects together the paths $\omega$ which yield a given value $\Sigma$ of $\int \mathrm{d} Q_{2} / T$, one obtains a "transient" form of the Gallavotti-Cohen relation:

$$
\frac{P(\Sigma)}{P(-\Sigma)}=\mathrm{e}^{\Sigma} .
$$

The Gallavotti-Cohen relation follows in the limit of long times, by setting

$$
P(\Sigma) \simeq \mathrm{e}^{t \Lambda(\sigma)}
$$

where

$$
\Sigma=t \sigma
$$

and where $\Lambda(\sigma)$ (implicitly defined by eq. (39) itself) is the large-deviation function for the entropy production rate. 


\section{ENTROPY PRODUCTION AND HEAT FLOW}

We provide here the physical interpretation of the quantity $\mathrm{d} Q_{2}(t)$. Let us define the local entropy

$$
s(x, t)=-\ln P(x, t) .
$$

The Shannon entropy of the system is thus given by

$$
S(t) \equiv\langle s(x, t)\rangle=-\int \mathrm{d} x P(x, t) \ln P(x, t) .
$$

The time derivative of this quantity is given by

$$
\begin{aligned}
\frac{\partial S(t)}{\partial t} & =-\int \mathrm{d} x\left\{\frac{\partial P(x, t)}{\partial t} \ln [P(x, t)]+P(x, t) \frac{\partial \ln P(x, t)}{\partial t}\right\} \\
& =-\int \mathrm{d} x \frac{\partial P(x, t)}{\partial t} \ln P(x, t) \\
& =\int \mathrm{d} x\left[\zeta T\left(\frac{\boldsymbol{p}}{T m}+\frac{\partial \ln P}{\partial \boldsymbol{p}}\right)^{2} P-\zeta \frac{\boldsymbol{p}}{m} \cdot\left(\frac{\boldsymbol{p}}{T m}+\frac{\partial \ln P}{\partial \boldsymbol{p}}\right) P\right],
\end{aligned}
$$

where the integral of the second term of eq. (43) vanishes because of the conservation of the pdf normalization. For the details of the calculations see the appendix, equation (A.1). On the other hand we have

$$
\left\langle\left.\frac{\mathrm{d} s(x(t), t)}{\mathrm{d} t}\right|_{x(t)=x}\right\rangle_{\eta}=\int \mathrm{d} x P(x, t)\left[\zeta T\left(\frac{\boldsymbol{p}}{T m}+\frac{\partial \ln P}{\partial \boldsymbol{p}}\right)^{2}-\zeta \frac{\boldsymbol{p}}{m} \cdot\left(\frac{\boldsymbol{p}}{T m}+\frac{\partial \ln P}{\partial \boldsymbol{p}}\right)\right] .
$$

where $\langle\cdots\rangle_{\eta}$ indicates the average over the pdf $P(x, t)$ and over the realizations of the process. For the details of the calculations see the appendix, equations A.2 A.6. Thus we obtain the result

$$
\frac{\mathrm{d} S(t)}{\mathrm{d} t}=\left\langle\left.\frac{\mathrm{d} s(x(t), t)}{\mathrm{d} t}\right|_{x(t)=x}\right\rangle_{\eta} .
$$

Let us now define the quantity

$$
\begin{aligned}
\mathrm{d} Q_{\mathrm{p}} & =\mathrm{d} Q_{2}+T\left(\frac{\partial s}{\partial \boldsymbol{r}} \cdot \mathrm{d} \boldsymbol{r}+\frac{\partial s}{\partial \boldsymbol{p}} \cdot \mathrm{d} \boldsymbol{p}\right) \\
& =-\left.\frac{\partial E}{\partial x}\right|_{x(t)} \mathrm{d} x(t)+\boldsymbol{f}_{\mu(t)} \cdot \frac{\boldsymbol{p}(t)}{m} \mathrm{~d} t+T\left(\frac{\partial s}{\partial \boldsymbol{r}} \cdot \mathrm{d} \boldsymbol{r}+\frac{\partial s}{\partial \boldsymbol{p}} \cdot \mathrm{d} \boldsymbol{p}\right)
\end{aligned}
$$

We have

$$
\begin{aligned}
\left\langle\left.\frac{\mathrm{d} Q_{\mathrm{p}}(x(t), t)}{\mathrm{d} t}\right|_{x(t)=x}\right\rangle_{\boldsymbol{\eta}} & =\left\langle\zeta\left(\frac{\boldsymbol{p}}{m}+T \frac{\partial \ln P}{\partial \boldsymbol{p}}\right)^{2}\right\rangle \\
& =T \frac{\mathrm{d} S(x, t)}{\mathrm{d} t}+\zeta \frac{\boldsymbol{p}}{m} \cdot\left(\frac{\boldsymbol{p}}{m}+T \frac{\partial \ln P}{\partial \boldsymbol{p}}\right) .
\end{aligned}
$$

The last equation indicates that the average of $\dot{Q}_{\mathrm{p}} / T$ is a non negative quantity, and can thus be interpreted as the entropy production rate of the process. Furthermore, rearranging eq. (49) we have

$$
\mathrm{d} Q_{\mathrm{p}}-\mathrm{d} Q_{2}=T\left(\frac{\partial s}{\partial \boldsymbol{r}} \cdot \mathrm{d} \boldsymbol{r}+\frac{\partial s}{\partial \boldsymbol{p}} \cdot \mathrm{d} \boldsymbol{p}\right) .
$$

Since one finds

$$
\left\langle\left.\frac{\mathrm{d} s(x(t), t)}{\mathrm{d} t}\right|_{x(t)=x}\right\rangle_{\eta}=\left\langle\frac{\partial s}{\partial \boldsymbol{r}} \cdot \mathrm{d} \boldsymbol{r}+\frac{\partial s}{\partial \boldsymbol{p}} \cdot \mathrm{d} \boldsymbol{p}\right\rangle_{\eta},
$$

(see appendix, eqs. (A.2)- A.3)), exploiting eq. (47), one ends up with

$$
\Delta S \equiv \int_{t_{0}}^{t} \frac{\mathrm{d} S(t)}{\mathrm{d} t}=\frac{1}{T} \int_{t_{0}}^{t}\left\langle\mathrm{~d} Q_{\mathrm{p}}-\mathrm{d} Q_{2}\right\rangle_{\eta}
$$

Thus, we can interpret the quantity $\mathrm{d} Q_{2} / T$ as the entropy flow into the heat reservoir. 


\section{FLUCTUATION RELATIONS AND THE ONSAGER-MACHLUP FUNCTIONAL}

Following the approach outlined in [10], and introducing the variable $\gamma^{(p)}$ conjugate to $\boldsymbol{p}$ and $\gamma^{(r)}$ conjugate to $\boldsymbol{r}$, we can express the functional $\Psi$ as

$$
\Psi\left(x, t ; x_{0}, t_{0} ; \mu, a\right)=\lim _{\mathcal{N} \rightarrow \infty} \int \prod_{k=1}^{\mathcal{N}} \frac{\mathrm{d} \gamma_{k} \mathrm{~d} x_{k}}{(2 \pi \mathrm{i})^{2 d}} \delta\left(x-x_{\mathcal{N}}\right) \exp \left\{\sum_{k=1}^{\mathcal{N}}\left[\gamma_{k}\left(x_{k}-x_{k-1}\right)+\left(L\left(\gamma_{k}, x_{k-1}, t_{k-1}\right)+a\left(x_{k-1}, t_{k-1}\right)\right) \Delta t\right]\right\}
$$

where

$$
\begin{aligned}
\Delta t & =\frac{t-t_{0}}{\mathcal{N}} ; \\
t_{k} & =t_{0}+k \Delta t \\
\gamma x & =\gamma^{(p)} \cdot \boldsymbol{p}+\gamma^{(r)} \cdot \boldsymbol{r} ; \\
L(\gamma, x, t) & =\gamma^{(r)} \cdot\left(-\frac{\boldsymbol{p}}{m}\right)+\gamma^{(p)} \cdot\left[\zeta \frac{\boldsymbol{p}}{m}+\frac{\partial U_{\mu(t)}}{\partial \boldsymbol{r}}-\boldsymbol{f}_{\mu(t)}+\zeta T \gamma^{(p)}\right] .
\end{aligned}
$$

In the continuum limit, equation (53) becomes

$$
\Psi\left(x, t ; x_{0}, t_{0} ; \mu, a\right)=\int_{x\left(t_{0}\right)=x_{0}}^{x(t)=x} \mathcal{D} \gamma \mathcal{D} \omega \mathrm{e}^{\mathcal{S}[\gamma, \omega, a]}
$$

where

$$
\mathcal{S}[\gamma, \omega, a]=\int_{t_{0}}^{t} \mathrm{~d} t^{\prime}\left[\gamma\left(t^{\prime}\right) \dot{x}\left(t^{\prime}\right)+L\left(\gamma\left(t^{\prime}\right), x\left(t^{\prime}\right), t^{\prime}\right)+a\left(x\left(t^{\prime}\right), t^{\prime}\right)\right]
$$

is the "action" associated to the given path $\omega$. From eq. (34), taking into account eq. (35), we obtain

$$
\Psi\left(\mathcal{I} x_{0}, t ; \mathcal{I} x, t_{0} ; \tilde{\mu}, \tilde{a}+w\right)=\int_{x\left(t_{0}\right)=\mathcal{I} x}^{x(t)=\mathcal{I} x_{0}} \mathcal{D} \gamma \mathcal{D} \tilde{\omega} \mathrm{e}^{\mathcal{S}[\gamma, \tilde{\omega}, \tilde{a}+w]}=\int_{x\left(t_{0}\right)=x_{0}}^{x(t)=x} \mathcal{D} \gamma \mathcal{D} \omega \mathrm{e}^{\widetilde{\mathcal{S}}[\gamma, \omega, a]},
$$

where

$$
\widetilde{\mathcal{S}}[\gamma, \omega, a]=\mathcal{S}[\gamma, \omega, a]+\frac{1}{T} \int_{t_{0}}^{t} \mathrm{~d} t^{\prime}\left(\left.\frac{\partial E}{\partial x}\right|_{x\left(t^{\prime}\right)} \dot{x}\left(t^{\prime}\right)-\boldsymbol{f}_{\mu\left(t^{\prime}\right)} \cdot \frac{\boldsymbol{p}\left(t^{\prime}\right)}{m}\right) .
$$

The second term on the rhs of the last equation corresponds to $-\int_{t_{0}}^{t} \mathrm{~d} Q_{2}$, where $\mathrm{d} Q_{2}$ is defined by eq. (35), along the given path $\omega$.

We can obtain an explicit expression for the probabilities of the path by performing the gaussian functional integral over $\gamma$. By taking $a(x, t)=0$ from eq. (12) and eq. 53, we obtain that the probability of a given path $\omega$ is given by

$$
\mathcal{P}[\omega]=\lim _{\mathcal{N} \rightarrow \infty} \int \prod_{k=1}^{\mathcal{N}}\left[\frac{\mathrm{d} \gamma_{k}}{(2 \pi \mathrm{i})^{2 d}} \mathrm{e}^{\gamma_{k}\left(x_{k}-x_{k-1}\right)+L\left(\gamma_{k}, x_{k-1}, t_{k-1}\right) \Delta t}\right] .
$$

If in last equation we substitute the expression for $L$ as given by eq. (57), we obtain

$$
\begin{aligned}
\mathcal{P}[\omega]= & \lim _{\mathcal{N} \rightarrow \infty} \int \prod_{k=1}^{\mathcal{N}}\left[\frac{\mathrm{d} \boldsymbol{\gamma}_{k}^{(r)}}{(2 \pi \mathrm{i})^{d}} \frac{\mathrm{d} \boldsymbol{\gamma}_{k}^{(p)}}{(2 \pi \mathrm{i})^{d}}\right] \exp \left\{\sum _ { k = 1 } ^ { \mathcal { N } } \left[\boldsymbol{\gamma}_{k}^{(r)}\left(\Delta \boldsymbol{r}_{k}-\frac{\boldsymbol{p}_{k}}{m} \Delta t\right)+\Delta t \zeta T\left(\gamma_{k}^{(p)}\right)^{2}\right.\right. \\
& \left.\left.+\boldsymbol{\gamma}^{(p)}\left[\Delta \boldsymbol{p}_{k}+\Delta t\left(\zeta \frac{\boldsymbol{p}_{k}}{m}+\left.\frac{\partial U_{\mu\left(t_{k}\right)}}{\partial \boldsymbol{r}}\right|_{\boldsymbol{r}_{k}}-\boldsymbol{f}_{\mu\left(t_{k}\right)}\right)\right]\right]\right\} \\
\propto & \lim _{\mathcal{N} \rightarrow \infty} \prod_{k=1}^{\mathcal{N}} \delta\left(\Delta \boldsymbol{r}_{k}-\frac{\boldsymbol{p}_{k}}{m} \Delta t\right) \exp \left\{-\frac{1}{4 \zeta T \Delta t}\left[\Delta \boldsymbol{p}_{k}+\Delta t \boldsymbol{G}\left(\boldsymbol{p}_{k}, \boldsymbol{r}_{k}, t_{k}\right)\right]^{2}\right\} .
\end{aligned}
$$

where

$$
\boldsymbol{G}\left(\boldsymbol{p}_{k}, \boldsymbol{r}_{k}, t_{k}\right)=\zeta \frac{\boldsymbol{p}_{k}}{m}+\left.\frac{\partial U_{\mu\left(t_{k}\right)}}{\partial \boldsymbol{r}}\right|_{\boldsymbol{r}_{k}}-\boldsymbol{f}_{\mu\left(t_{k}\right)}
$$


Equation (63) indicates that the probability of a given path is non-zero only if the path satisfies

$$
p=m \dot{r},
$$

as expected. Some care has to be taken in passage to the continuum limit in eq. (63): the argument of the exponential in this equation reads

$$
\sum_{k=1}^{\mathcal{N}}-\frac{\Delta t}{4 \zeta T}\left[\frac{\Delta \boldsymbol{p}_{k}}{\Delta t}+\boldsymbol{G}\left(\boldsymbol{p}_{k}, \boldsymbol{r}_{k}, t_{k}\right)\right]^{2}
$$

We consider separately the large $\mathcal{N}$ limit of each of the terms appearing in this sum: the first and the second term read

$$
\begin{aligned}
& \lim _{\mathcal{N} \rightarrow \infty}\left(-\frac{\Delta t}{4 \zeta T}\right) \sum_{k=1}^{\mathcal{N}}\left(\frac{\Delta \boldsymbol{p}_{k}}{\Delta t}\right)^{2}=-\frac{1}{4 \zeta T} \int_{t_{0}}^{t} \mathrm{~d} t^{\prime}\left(\dot{\boldsymbol{p}}\left(t^{\prime}\right)\right)^{2}, \\
& \lim _{\mathcal{N} \rightarrow \infty}\left(-\frac{\Delta t}{4 \zeta T}\right) \sum_{k=1}^{\mathcal{N}}\left[\boldsymbol{G}\left(\boldsymbol{p}_{k}, \boldsymbol{r}_{k}, t_{k}\right)\right]^{2}=-\frac{1}{4 \zeta T} \int_{t_{0}}^{t} \mathrm{~d} t^{\prime} \boldsymbol{G}^{2}\left(\boldsymbol{p}, \boldsymbol{r}, t^{\prime}\right),
\end{aligned}
$$

respectively. The expression appearing on the rhs of eq. (67) is of course only formal, since the weight of the functional integral is concentrated on functions that are continuous but not differentiable. The double product reads

$$
\lim _{\mathcal{N} \rightarrow \infty}\left(-\frac{1}{2 \zeta T}\right) \sum_{k=1}^{\mathcal{N}}\left[\left(\boldsymbol{p}_{k}-\boldsymbol{p}_{k-1}\right) \cdot \boldsymbol{G}\left(\boldsymbol{p}_{k}, \boldsymbol{r}_{k}, t_{k}\right)\right] \equiv-\frac{1}{2 \zeta T}(I) \int \mathrm{d} \boldsymbol{p} \cdot \boldsymbol{G}(\boldsymbol{p}, \boldsymbol{r}, t),
$$

and, as discussed in ref. [11], the value of the integral depends on the discretization scheme: we have consequently used the prefix (I) indicating that the integral is the continuum limit of an Itō sum. We can express this quantity in a more convenient way by writing, in analogy with Itō's formula (see eqs. (17)-(19) in ref. [11] and references therein)

$$
\text { (I) } \int \mathrm{d} \boldsymbol{p} \cdot \boldsymbol{G}(\boldsymbol{p}, \boldsymbol{r}, t)=\int_{t_{0}}^{t} \boldsymbol{G}\left(\boldsymbol{p}\left(t^{\prime}\right), \boldsymbol{r}\left(t^{\prime}\right), t^{\prime}\right) \cdot \dot{\boldsymbol{p}}\left(t^{\prime}\right) \mathrm{d} t^{\prime}-\left.\zeta T \int_{t_{0}}^{t} \mathrm{~d} t^{\prime} \sum_{\alpha=1}^{d} \frac{\partial G_{\alpha}}{\partial p_{\alpha}}\right|_{\boldsymbol{p}\left(t^{\prime}\right), \boldsymbol{r}\left(t^{\prime}\right), t^{\prime}} .
$$

Thus, substituting eqs. (67), (68) and (70), into eq. (63), in the continuum limit eq. (62) becomes

$$
\mathcal{P}(\omega) \propto \exp \left[-\frac{1}{4 \zeta T} \int_{t_{0}}^{t}\left(m \ddot{\boldsymbol{r}}\left(t^{\prime}\right)+\zeta \dot{\boldsymbol{r}}+\left.\frac{\partial U_{\mu\left(t^{\prime}\right)}}{\partial \boldsymbol{r}}\right|_{\boldsymbol{r}\left(t^{\prime}\right)}-\boldsymbol{f}_{\mu\left(t^{\prime}\right)}\right)^{2} \mathrm{~d} t^{\prime}+\frac{3 \zeta}{2 m}\left(t-t_{0}\right)\right] .
$$

These expressions correspond to those obtained by Onsager and Machlup [12] for the harmonically bound Brownian particle. Note that the linear term appearing in the exponential of eq. (71), and which arises from eq. (70), can be "hidden" in the normalization of the probability function $\mathcal{P}(\omega)$ as long as one deals with a force $G$ linear in $\boldsymbol{p}$.

From eq. (71), we are able to recover eq. (34) directly. In fact, for the inverse path $\tilde{\omega}$, eq. (71) reads

$$
\mathcal{P}(\tilde{\omega}) \propto \exp \left[-\frac{1}{4 \zeta T} \int_{t_{0}}^{t}\left(m \ddot{\boldsymbol{r}}\left(t^{\prime}\right)-\zeta \dot{\boldsymbol{r}}\left(t^{\prime}\right)+\left.\frac{\partial U_{\mu\left(t^{\prime}\right)}}{\partial \boldsymbol{r}}\right|_{\boldsymbol{r}\left(t^{\prime}\right)}-\boldsymbol{f}_{\mu\left(t^{\prime}\right)}\right)^{2} \mathrm{~d} t^{\prime}+\frac{3 \zeta}{2 m}\left(t-t_{0}\right)\right],
$$

and thus we have, after straightforward manipulations,

$$
\begin{aligned}
\frac{\mathcal{P}(\omega)}{\mathcal{P}(\tilde{\omega})} & =\exp \left\{\frac{1}{T} \int_{t_{0}}^{t} \mathrm{~d} t^{\prime}\left[-\frac{\boldsymbol{p}\left(t^{\prime}\right)}{m} \cdot\left(\dot{\boldsymbol{p}}\left(t^{\prime}\right)+\left.\frac{\partial U_{\mu(t)}}{\partial \boldsymbol{r}}\right|_{\boldsymbol{r}\left(t^{\prime}\right)}-f_{\mu\left(t^{\prime}\right)}\right)\right]\right\} \\
& =\int_{x_{0}}^{x} \frac{\mathrm{d} Q_{2}}{T}
\end{aligned}
$$

where $\mathrm{d} Q_{2}$ is given by eq. (35).

While eq. (71) is well known, we wish to emphasize the relation of the entropy flow along a given trajectory $\omega$, with the generalized Onsager-Machlup functional for that trajectory, expressed by eq. (73). 


\section{DISCUSSION}

We have obtained a compact derivation of the relation between the probability of a given path in phase space and that of its time-reversed image in the specular manipulation protocol. The ratio of the probabilities has been interpreted as the entropy flow into the heat bath, by a suitable generalization of the expression of the heat flow. Different fluctuation relations can be derived from this key equality by simple manipulations. The approach can be easily applied to more complex systems, and to situations in which the energy function is not invariant under time reversal, due, e.g., to the presence of magnetic fields. We have also shown how the basic relation directly follows from the expression of the path probability first established by Onsager and Machlup [12] for the harmonically bound particle. We hope that the present work can be useful in the investigation of more and more general aspects of the nonequilibrium thermodynamics of small systems.

\section{Acknowledgments}

This research was partially supported by MIUR-PRIN 2004. We are grateful to G. E. Crooks for suggesting us some corrections and for pointing out to us ref. [11].

\section{APPENDIX: DERIVATION OF THE EXPRESSIONS FOR THE TIME DERIVATIVE OF THE ENTROPY}

Let us first derive eq. (45). After substitution of equations (2) and (3) into eq. (44), we obtain

$$
\begin{aligned}
\frac{\mathrm{d} S(t)}{\mathrm{d} t} & =-\int \mathrm{d} x \ln P\left\{\frac{\partial}{\partial \boldsymbol{r}} \cdot\left(-\frac{\boldsymbol{p}}{m} P\right)+\frac{\partial}{\partial \boldsymbol{p}} \cdot\left[\left(\zeta \frac{\boldsymbol{p}}{m}+\frac{\partial U_{\mu}}{\partial \boldsymbol{r}}-\boldsymbol{f}_{\mu}\right) P+\zeta T \frac{\partial}{\partial \boldsymbol{p}} P\right]\right\} \\
& =\int \mathrm{d} x\left\{\frac{\partial \ln P}{\partial \boldsymbol{r}} \cdot\left(-\frac{\boldsymbol{p}}{m} P\right)+\frac{\partial \ln P}{\partial \boldsymbol{p}} \cdot\left[\left(\zeta \frac{\boldsymbol{p}}{m}+\frac{\partial U_{\mu}}{\partial \boldsymbol{r}}-\boldsymbol{f}_{\mu}\right) P+\zeta T \frac{\partial}{\partial \boldsymbol{p}} P\right]\right\} \\
& =\int \mathrm{d} x\left[P \zeta \frac{\boldsymbol{p}}{m} \cdot \frac{\partial \ln P}{\partial \boldsymbol{p}}+\zeta T P\left(\frac{\partial \ln P}{\partial \boldsymbol{p}}\right)^{2}\right] \\
& =\int \mathrm{d} x\left[\zeta T\left(\frac{\boldsymbol{p}}{T m}+\frac{\partial \ln P}{\partial \boldsymbol{p}}\right)^{2}-\frac{\zeta}{T} \frac{\boldsymbol{p}}{m} \cdot\left(\frac{\boldsymbol{p}}{m}+\frac{\partial \ln P}{\partial \boldsymbol{p}}\right)\right] P(x, t),
\end{aligned}
$$

as can be checked by some manipulation. Equation (A.1) corresponds thus to eq. (45).

We can now prove eq. $(46)$. We have

$$
\left\langle\left.\frac{\mathrm{d} s(x(t), t)}{\mathrm{d} t}\right|_{x(t)=x}\right\rangle_{\boldsymbol{\eta}}=\left\langle\left.\left[\frac{\partial s(x(t), t)}{\partial \boldsymbol{r}} \cdot \dot{\boldsymbol{r}}+\frac{\partial s(x(t), t)}{\partial \boldsymbol{p}} \cdot \dot{\boldsymbol{p}}+\frac{\partial s(x(t), t)}{\partial t}\right]\right|_{x(t)=x}\right\rangle_{\boldsymbol{\eta}} .
$$

The last term on the rhs is independent of the path, and its average vanishes because of the normalization. Thus we are left with

$$
\left\langle\left.\frac{\mathrm{d} s(x(t), t)}{\mathrm{d} t}\right|_{x(t)=x}\right\rangle_{\boldsymbol{\eta}}=\int \mathrm{d} x P(x, t)\left\{\frac{\partial s(x, t)}{\partial \boldsymbol{r}} \cdot[\dot{\boldsymbol{r}}]_{\boldsymbol{\eta}}+\frac{\partial s(x, t)}{\partial \boldsymbol{p}} \cdot[\dot{\boldsymbol{p}}]_{\boldsymbol{\eta}}\right\},
$$

where $[\cdots]_{\eta}$ is the average over the process realization alone, constrained by the final state $x(t)=x$. For the process described by eqs. (2) and (3), one obtains

$$
\begin{aligned}
{[\dot{\boldsymbol{r}}]_{\boldsymbol{\eta}} } & =\frac{\boldsymbol{p}}{m} \\
{[\dot{\boldsymbol{p}}]_{\boldsymbol{\eta}} } & =-\zeta \frac{\boldsymbol{p}}{m}-\frac{\partial U_{\mu}}{\partial \boldsymbol{r}}+\boldsymbol{f}_{\mu}-\zeta T \frac{\partial \ln P(x, t)}{\partial \boldsymbol{p}}
\end{aligned}
$$

Hence, eq. A.3 becomes

$$
\begin{aligned}
\left\langle\left.\frac{\mathrm{d} s(x(t), t)}{\mathrm{d} t}\right|_{x(t)=x}\right\rangle_{\boldsymbol{\eta}} & =-\int \mathrm{d} x P\left\{\frac{\partial \ln P}{\partial \boldsymbol{r}} \cdot \frac{\boldsymbol{p}}{m}+\frac{\partial \ln P}{\partial \boldsymbol{p}} \cdot\left[-\zeta \frac{\boldsymbol{p}}{m}-\frac{\partial U_{\mu}}{\partial \boldsymbol{r}}+\boldsymbol{f}_{\mu}-\zeta T \frac{\partial \ln P}{\partial \boldsymbol{p}}\right]\right\} \\
& =\int \mathrm{d} x P \frac{\partial \ln P}{\partial \boldsymbol{p}} \cdot\left[\zeta \frac{\boldsymbol{p}}{m}+\zeta T \frac{\partial \ln P}{\partial \boldsymbol{p}}\right] \\
& =\int \mathrm{d} x P\left[\zeta T\left(\frac{\boldsymbol{p}}{T m}+\frac{\partial \ln P}{\partial \boldsymbol{p}}\right)^{2}-\zeta \frac{\boldsymbol{p}}{m} \cdot\left(\frac{\boldsymbol{p}}{T m}+\frac{\partial \ln P}{\partial \boldsymbol{p}}\right)\right],
\end{aligned}
$$


which corresponds to eq. 46.

[1] D. J. Evans and D. J. Searles, Equilibrium microstates which generate second law violating steady states, Phys. Rev. E 50, 1645 (1994).

[2] G. Gallavotti and E.G.D. Cohen, Dynamical ensemble in a stationary state, J. Stat Phys. 80, 931 (1995).

[3] C. Jarzynski, Nonequilibrium equality for free energy differences, Phys. Rev. Lett. 78, 2690 (1997).

[4] T. Hatano and S. Sasa, Steady-state thermodynamics of Langevin systems, Phys. Rev. Lett. 86, 3463 (2000).

[5] U. Seifert, Entropy production along a stochastic trajectory and an integral fluctuation theorem, Phys. Rev. Lett. 95, 040602 (2005).

[6] This point has been emphasized in particular by R. D. Astumian, The unreasonable effectiveness of equilibrium-like theory for interpreting non-equilibrium experiments, Am. J. of Physics., in press.

[7] J. Kurchan, Fluctuation theorem for stochastic dynamics, J. Phys. A: Math. Gen. 31, 3719 (1998).

[8] G. E. Crooks, Path-ensemble averages in systems driven far from equilibrium, Phys. Rev. E 61, 2361 (2000).

[9] K. Sekimoto, Kinetic characterization of heat bath and the energetics of thermal ratchet models, J. Phys. Soc. Japan, 66, 1234 (1996).

[10] A. Imparato, L. Peliti, Work distribution and path integrals in general mean-field systems, Europhysics Letters 70, 740 (2005); A. Imparato, L. Peliti, Work-probability distribution in systems driven out of equilibrium, Phys. Rev. E 72, 046114 (2005).

[11] K. L. Hunt, J. Ross, Path integral solutions of stochastic equations for nonlinear irreversible processes: The uniqueness of the thermodynamic Lagrangian, J. Chem. Phys. 75, 976 (1981).

[12] L. Onsager and S. Machlup, Fluctuations and irreversible processes, Phys. Rev. 91, 1505 (1952); S. Machlup and L. Onsager, Fluctuations and irreversible process. II. Systems with kinetic energy, Phys. Rev. 91, 1512 (1952). 